\title{
Endocrine System Disorder
}

National Cancer Institute

\section{Source}

National Cancer Institute. Endocrine System Disorder. NCI Thesaurus. Code C3009.

A non-neoplastic or neoplastic disorder that affects the endocrine system.

Representative examples of non-neoplastic disorders include diabetes mellitus,

hyperthyroidism, and adrenal gland insufficiency. Representative examples of neoplastic

disorders include carcinoid tumor, neuroendocrine carcinoma, and pheochromocytoma. 